Кузьмов А. В. Штерн М. Б.

\title{
УМЕНЬШЕНИЕ ДАВЛЕНИЯ ПУТЕМ ВРАЩЕНИЯ ПРЕСС-ИНСТРУМЕНТА ПРИ РАДИАЛЬНОМ ПРЕССОВАНИИ ТРУБЧАТЫХ ЗАГОТОВОК ИЗ ПОРОШКОВ МЕТАЛЛОВ
}

Методы обработки давлением материалов на основе порошков черных и цветных металлов являются одной из составляющих технологий современного машиностроения. Низкая металлоемкость, высокая размерная точность, а главное, возможность совмещения высоких механических свойств с функциональными характеристиками порошкового изделия определяет высокую конкурентоспособность данных методов и их распространение в мировой практике машиностроения. Традиционно наибольшее распространение получили схемы прессования в замкнутых неподвижных пресс-формах, то есть схемы одноосного деформирования. С другой стороны, известно, что наличие сдвиговых компонент деформации в определенной степени уменьшает рабочее давление прессования $[1,2]$.

В то же время применение схем деформирования, обеспечивающих наличие сдвиговых деформаций, находит в настоящее время широкое применение при обработке давлением компактных материалов. При этом, наряду с традиционными технологиями штамповки, существуют методы обработки, основной целью которых является накопление деформаций в заготовках, а не изменение их формы. К таким методам относятся винтовая экструзия [3], равноканальная угловая экструзия $[4,5]$, кручение под давлением $[4,6]$ и другие. В результате использования таких технологических процессов можно получить ультрамелкозернистую структуру обрабатываемого материала, который приобретает при этом качественно новые свойства.

В последнее время ведутся активные исследования по использованию этих технологий для обработки порошковых заготовок $[7,8,9]$. Наличие сдвиговых деформаций приводит к благоприятной ориентации металлических зерен, неметаллических включений и пор, появлению текстуры. Важной задачей при этом является возможность снизить энергосиловые параметры процесса и, в результате, повысить устойчивость деформирующего инструмента.

Следует отметить, что теория пластичности сжимаемых материалов прогнозирует уменьшение давления прессования при мгновенном появлении сдвиговых деформаций [1]. Однако в ходе длительного технологического процесса наличие дополнительных сдвиговых деформаций вызывает увеличение накопленной деформации и деформационного упрочнения, что приводит к противоположному эффекту увеличения давления прессования. Поэтому вопрос о целесообразности применения нестандартных схем прессования порошков со сдвиговыми деформациями, кроме анализа целесообразности усложнения технологического оборудования, требует также исследования влияния деформационного упрочнения. Такое исследование проводилось одним из авторов в работе [2] в которой схема с вращением пуансона при прессовании в закрытой пресс-форме исследовалась путем вычислительного моделирования методом конечных элементов. В этой работе было рассмотрено несколько конкретных случаев кинематической схемы и реологических параметров материала. Однако рекомендаций как в общем случае оценивать подобные схемы прессования дано не было Поэтому возникает вопрос, нельзя ли исследовать влияние сдвиговых деформаций на давление прессования с учетом деформационного упрочнения материала в аналитическом виде для однородных напряженно-деформированных состояний, подобно тому, как это сделано для стандартных схем прессования в [10]. Это позволило бы, по крайней мере, на качественном уровне, оценивать подобные схемы прессования со сдвиговыми деформациями. 
Схема прессования и реологическая модель материала.

Оказывается такой простой для анализа схемой может стать радиальное или осевое прессования трубчатых заготовок совмещенное с вращением внутренней и внешней стенок пресс-формы с разной угловой скоростью $\omega_{1}$ и $\omega_{2}$. В данной работе рассматривается случай радиального прессования (рис. 1). При этом предполагается полное прилипание материала прессовки к внутренней и внешней стенкам. Ненулевыми компонентами тензора скоростей деформаций являются только $e_{r r}$ и $e_{r \phi}$. При этом $e_{r \phi}=\frac{\omega_{1}-\omega_{2}}{2 \ln \left(r_{1} / r_{2}\right)}$, где $r_{1}$ и $r_{2}$ - соответственно внешний и внутренний радиусы трубчатой заготовки.

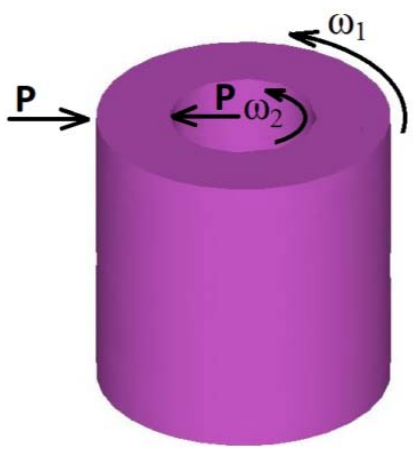

Рис. 1. Радиальное прессование трубчатых заготовок, совмещенное с вращением боковых стенок с разной угловой скоростью

В качестве определяющих соотношений берется пластическая модель на основе ассоциированного закона пластического течения $e_{i j}=\lambda \frac{\partial F}{\sigma_{i j}} ; \lambda>0$ и поверхности нагружения $F$ в виде:

$$
\begin{aligned}
& F\left(\sigma_{i j}\right)=\frac{p^{2}}{\psi}+\frac{\tau^{2}}{\varphi}-(1-\theta) \tau_{0}^{2} ; \quad \psi=\frac{2(1-\theta)^{3}}{3 \theta} ; \quad \varphi=(1-\theta)^{2} ; \\
& p=\frac{1}{3} \sum_{i=1}^{3} \sigma_{i i} ; \quad \tau^{2}=\sum_{i, j=1}^{3}\left(\sigma_{i j}-p \delta_{i j}\right)^{2} ;
\end{aligned}
$$

где $p, \tau$ - первый и второй инварианты тензора напряжений соответственно, $\delta_{i j}$ символ Кронекера или единичный тензор, $\theta$ - пористость, то есть объёмная доля пустот в материале, $\tau_{0}$ - предел текучести «твердой фазы», то есть материала каркаса пористого тела. Деформационное упрочнение описывается уравнением:

$$
\begin{aligned}
& \tau_{0}=a+b \omega^{\alpha} ; \quad \frac{d \omega}{d t}=\frac{1}{\sqrt{1-\theta}} \sqrt{\psi e^{2}+\varphi \gamma^{2}} ; \\
& e=\sum_{i=1}^{3} e_{i i} ; \quad \gamma^{2}=\sum_{i, j=1}^{3}\left(e_{i j}-\frac{1}{3} e \delta_{i j}\right)^{2} ;
\end{aligned}
$$

где $a, b, \alpha$ - начальный предел текучести, коэффициент упрочнения и показатель упрочнения, соответственно, для материала порошка, $e, \gamma-$ первый и второй инварианты тензора скоростей деформаций соответственно, $\omega$ - накопленная деформация «твердой фазы».

Поверхности нагружения (1) будет соответствовать удельная диссипация: 


$$
D\left(e_{i j}\right)=\tau_{0} \sqrt{1-\theta} \sqrt{\psi e^{2}+\varphi \gamma^{2}},
$$

которая является также диссипативным потенциалом, то есть:

$$
\sigma_{i j}=\frac{\partial D}{\partial e_{i j}} .
$$

Из (3) и (4) с учетом равенств $\frac{\partial e}{\partial e_{i j}}=\delta_{i j}$ и $\frac{\partial \gamma}{\partial e_{i j}}=\frac{e_{i j}-\frac{1}{3} e \delta_{i j}}{\gamma}$ получим выражения для напряжений через скорости деформаций:

$$
\sigma_{i j}=\frac{\tau_{0} \sqrt{1-\theta}}{\sqrt{\psi e^{2}+\varphi \gamma^{2}}}\left[\left(\psi-\frac{\varphi}{3}\right) e \delta_{i j}+\varphi e_{i j}\right] \text {. }
$$

В случае ненулевых скоростей деформаций только $e_{r r}$ и $e_{r \phi}$ инварианты $e, \gamma$ примут вид $e=2 e_{r r} \gamma^{2}=\frac{2}{3} e_{r r}^{2}+2 e_{r \phi}^{2}$ и рабочее давление прессования $P=-\sigma_{r r}$, исходя из (5), запишется в виде

$$
P=-\sigma_{r r}=-\frac{\tau_{0} \sqrt{1-\theta}\left(2 \psi+\frac{\varphi}{3}\right) e_{r r}}{\sqrt{2} \sqrt{\left(2 \psi+\frac{\varphi}{3}\right) e_{r r}^{2}+\varphi e_{r \phi}^{2}}} .
$$

Выразив ч и фчерез пористость $\theta$ согласно (1) и с учетом $e_{r r}<0$ получим

$$
P=-\sigma_{r r}=\tau_{0} \sqrt{\frac{3}{2}} \frac{(1-\theta)^{\frac{3}{2}}}{\sqrt{\theta}} \frac{\sqrt{4-3 \theta}}{\sqrt{1+\frac{3 \theta}{4-3 \theta} K^{2}}} ; \quad K=\left|\frac{e_{r \phi}}{e_{r r}}\right| .
$$

Параметр $K$ является мерой дополнительных сдвиговых деформаций, возникающих в результате вращения друг относительно друга внутренней и внешней стенок пресс-формы. Кинематика рассматриваемой в этой работе схемы холодного осевого прессования трубчатых заготовок совмещенного с вращением внутренней и внешней стенок пресс-формы с разной угловой скоростью характеризуется одним лишь этим параметром $K$, в силу нечувствительности усилий припластической деформации к абсолютной величине скорости.

Поскольку в нашем случае пористость монотонно убывает по времени, то в качестве параметра, характеризующего ход процесса уплотнения, можно вместо времени взять пористость. Время и пористость связаны между собой уравнением неразрывности (сохранения массы):

$$
\frac{d \theta}{d t}=(1-\theta) e
$$

Тогда для накопленной деформации «твердой фазы» с учетом (2) и (8), а также выразив ч и $\varphi$ через пористость $\theta$ согласно (1), получим дифференциальное уравнение:

$$
\frac{d \omega}{d \theta}=-\frac{\sqrt{\frac{4-3 \theta}{3 \theta}+K^{2}}}{\sqrt{2(1-\theta)}}
$$


Общим решением этого уравнения является неопределенный интеграл, который может быть выражен через специальные функции - «эллиптические интегралы».

$$
E\{M, N\}=\int_{0}^{M} \sqrt{1-N^{2} \sin ^{2} Y} d Y
$$

Выпишем общее решение уравнения (9):

$$
\begin{aligned}
& F(\theta, K)=-\frac{1}{\sqrt{6}} \int \frac{\sqrt{4+3 \theta\left(K^{2}-1\right)}}{\sqrt{\theta} \sqrt{1-\theta}} d \theta= \begin{cases}F_{1}(\theta, K) ; & K>1 \\
-2 \sqrt{\frac{2}{3} \arcsin \sqrt{\theta} ;} & K=1 \\
F_{2}(\theta, K) ; & 0 \leq K<1\end{cases} \\
& F_{1}(\theta, K)=\sqrt{2\left(K^{2}-1\right)}\left[\sqrt{(1-\theta)} g_{1}(\theta)-\frac{1}{a} E\left\{\arcsin \left(g_{1}(\theta) / a\right), a\right\}\right] \\
& g_{1}(\theta)=\sqrt{\frac{3\left(K^{2}-1\right) \theta}{4+3 \theta\left(K^{2}-1\right)} ; \quad a=g_{1}(1)=\sqrt{\frac{3\left(K^{2}-1\right)}{3 K^{2}+1}}} \\
& F_{2}(\theta, K)=\sqrt{2\left(1-K^{2}\right)}\left[\frac{1}{b} E\left\{\arcsin \left(g_{2}(\theta) / b\right), b\right\}-\sqrt{\theta} g_{2}(\theta)\right]-\sqrt{\frac{2}{3}} \pi \\
& g_{2}(\theta)=\sqrt{\frac{3\left(1-K^{2}\right)(1-\theta)}{4-3 \theta\left(1-K^{2}\right)}} ; \quad b=g_{2}(0)=\frac{\sqrt{3\left(1-K^{2}\right)}}{2}
\end{aligned}
$$

Тогда решением уравнения (9) с начальным условием $\omega\left(\theta_{0}\right)=0$, где $\theta_{0}$ - начальная пористость, будет $\omega\left(\theta_{0}, \theta, K\right)=F(\theta, K)-F\left(\theta_{0}, K\right)$.

Влияние параметров материала и схемы прессования на рабочее давление.

Полученное таким образом аналитическое выражение для накопленной деформации после подстановки в (2) и (7) позволяет найти рабочее давление прессования тоже в замкнутом аналитическом виде $\sigma_{z z}=\sigma_{z z}\left(\theta_{0}, \theta, K, a, b, \alpha\right)$, где $\theta_{0}$ и $\theta$ - начальная и текущая пористость соответственно. На рис. 2 сравниваются кривые давление-плотность при наличии и отсутствии взаимного вращения боковых стенок для материала с параметрами упрочнения $b=5 a, \alpha=0.9$. Видно, что, начиная с определенного момента, взаимное вращение боковых стенок вызывает увеличение рабочего давления.

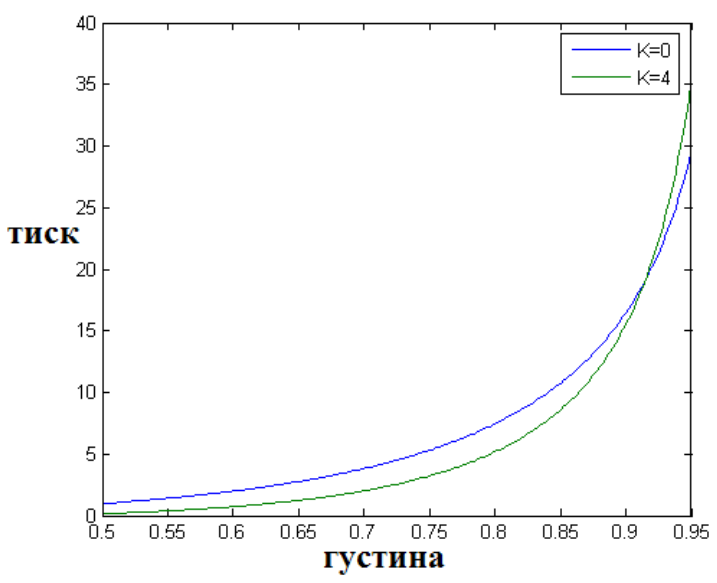

Рис. 2. Зависимость рабочего давления (нормированного пределом текучести «твердой фазы») от плотности 
Выражение $\sigma_{z z}=\sigma_{z z}\left(\theta_{0}, \theta, K, a, b, \alpha\right)$ было использовано для анализа целесообразности вращения пуансонов с точки зрения уменьшения рабочего давления. Пусть имеем порошковую заготовку из некоторого материала с начальной пористостью $\theta_{0}$, тогда $\sigma_{z z}$ зависит только от двух параметров $\theta$ и $K$. Поэтому имеет смысл отыскать такие значения $(\theta, K)$, при которых наблюдается уменьшение давления прессования по сравнению со схемой без сдвиговых деформаций. Для случая материала с параметрами упрочнения $b=5 a, \alpha=0.9$ и начальной пористостью 0.5 область таких значений $(\theta, K)$, то есть область целесообразности предложенной схемы, показана на рис. 3 (эта область зарисована серым цветом).

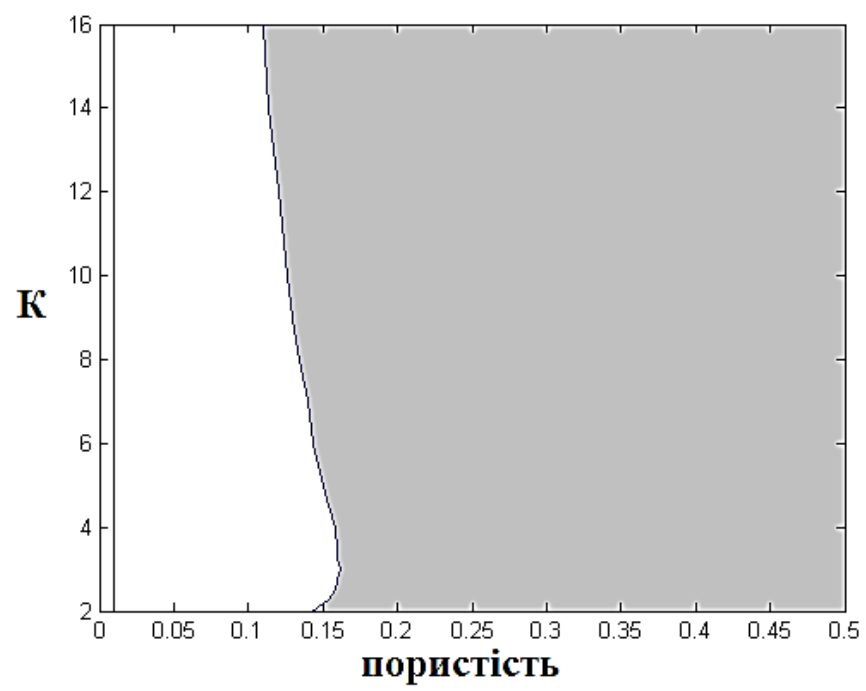

Рис. 3. Область целесообразности параметров $\theta, K$

Следует отметить, что как видно из (7) при значительных степенях уплотнения $(\theta \rightarrow 0)$ воздействие $K$ на рабочее давление значительно ослабляется, потому как видно из рис.3 для каждой величины $K$ существует такое предельное значение пористости, что для дальнейшего уплотнения сдвигать боковые стенки одну относительно другой в дальнейшем нецелесообразно. Однако такие предельные значения пористости, которые назовем в дальнейшем кривой целесообразности, существенно зависят не только от $K$, но и от начальной плотности заготовки. Это хорошо видно из рис. 4. где приведены кривые целесообразности для различных значений начальной пористости для того же материала $b=5 a, \alpha=0.9$.

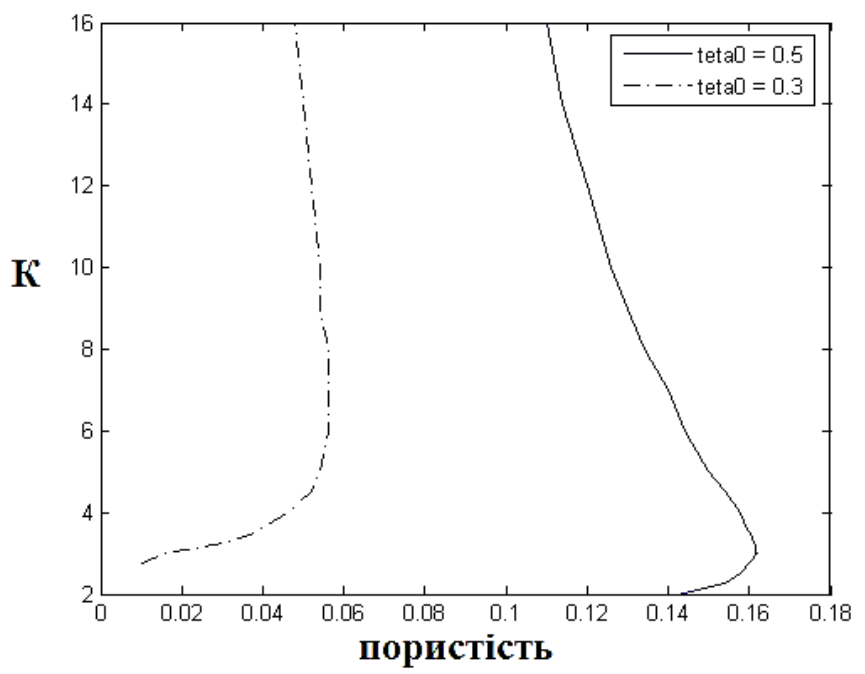

Рис. 4. Кривые целесообразности для различной начальной пористости $\theta_{0}$ 
Кривые целесообразности, естественно, очень существенно зависят от деформационного упрочнения материала (рис 5), причем как от коэффициента $b$, так и показателя упрочнения $\alpha$.

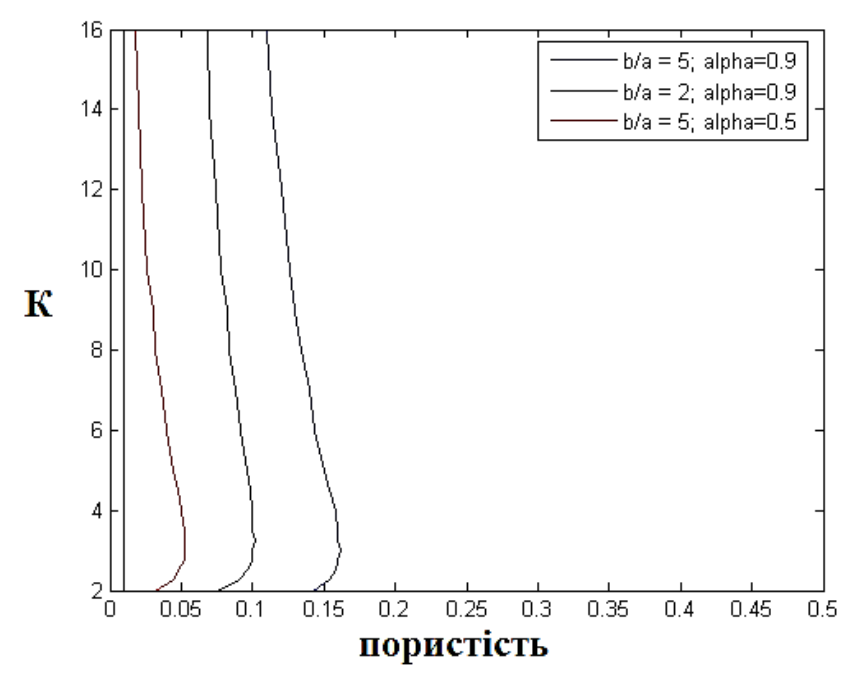

Рис. 5. Кривые целесообразности для различных случаев деформационного упрочнения с начальной пористости $\theta_{0}=0.5$

\section{ВЫВОДЫ}

На основе результатов, изложенных выше, установлено как влияет вращение матрицы относительно пуансона на зависимость между плотностью и давлением при холодном прессовании порошков в жесткой матрице. Важно, что решение данной задачи было получено в аналитической форме. Это решение позволяет иметь качественное представление как о процессах прессования порошков, совмещенных со сдвиговыми деформациями, так и о более сложных схемах с неоднородным напряженно-деформированным состоянием.

На начальной стадии прессования использование вращающихся матриц или пуансонов способствует снижению давления прессования. C возрастанием степени уплотнения, т. е. на поздних стадиях прессования наличие вращения приводит к возрастанию давления прессования. Момент перехода от стадии уменьшения давления к стадии его увеличения существенно зависит от того, насколько упрочняемым является материал порошка. Проведенный анализ свидетельствует о том, что с точки зрения указанных результатов, вращение матрицы целесообразно использовать для порошков, материал которых ближе к идеальнопластическому.

Анализ зависимости между плотностью и давлением, приведенной на рис. 2 и рис. 3, свидетельствует о том, что вращение матрицы на протяжении всего процесса прессования целесообразно использовать лишь в том случае, когда нужно получить не полностью допресованную заготовку, в которой пористость должна быть более, чем порядка 0,15 , и определяется начальной пористостью.

В то же время, если предположить ограниченную в силу технологических причин относительную величину сдвиговых деформаций $K$, то анализ рис. 3 и рис. 4 свидетельствует о том, что зачастую целесообразнее вращать стенки матрицы только в конце процесса прессования. То есть в случаях значительного уплотнения от больших до малых пористостей вращать друг относительно друга стенки матрицы с самого начала прессования нецелесообразно.

Вопрос же о том, как оптимально синхронизировать вращение стенок и степень уплотнения заготовки, требует дополнительного исследования, выходящего за рамки этой работы. Эта задача является интересной темой для дальнейших исследований в затронутом направлении. 


\section{СПИСОК ИСПОЛЬЗОВАННОЙ ЛИТЕРАТУРЫ}

1. Штерн М. Б. Развитие теории прессования и пластического деформирования порошковых материалов. Порошковая металлургия. 1992. 9. С. 12-24.

2. Михайлов О. В., Штерн М. Б. Вплив крутіння пористих заготовок на їх ущільнення в закритому штампі. Математичні моделі і обчислювальний експеримент в матеріалознавстві. Київ: ІПМ ім. І. М. Францевича НАН України. 2013. 15. С. 81-85.

3. Бейгельзимер Я. Е., Варюхин В. Н., Орлов Д. В., Сынков С. Г. Винтовая экструзия - процесс накопления деформации. Донецк : ТЕАН, 2003. 85 с.

4. Валиев Р. 3., Александров И. В. Наноструктурные материалы, полученные интенсивной пластической деформацией. Москва : Логос, 2000. 271 с.

5. Сегал В. М., Резников В. И., Копылов В. И., Павлик Д. А., Малышев В. Ф. Процессы пластического структурообразования металлов. Минск : Навука и тэхника, 1994. 232 с.

6. Бриджмен П. В. Исследование больших пластических деформаций и разрыва. Москва : Изд-во иностранной литературы, 1955. 444 с.

7. Varyukhin V.N., Beygelsimer Y.Y., Synkov S.G. etc. Consolidation of amorphous A186Ni6Go2Gd6 melt spun ribbons by twist extrusion. Material Science Forum. 2006. 503-504, pp. 699-704.

8. Xiang S., Matsuki K., Tahatsuju N., Tokizawa M., Yokote T., Kusui J., Yokoi K. Microstructure and mechanical properties of PM 2024Al - 3Fe - 5Ni alloy consolidated by a new process, equal channel angular pressing. Journal of Materials Science Letters. 1997. 16, pp. 1725-1727.

9. Xia K., Wu X. Back pressure equal channel angular consolidation of pure Al particles. Scripta Materialia. 2005. 53, pp. 1225-1229.

10. Штерн М. Б., Сердюк Г. Г. и др. Феноменологические теории прессования порошков. Київ : Наукова думка, 1982. 140 с.

\section{REFERENCES}

1. Stern M. B. Development of the theory of pressing and plastic deformation of powder materials. Powder Metallurgy. 1992, 9, pp. 12 - 24. (in Russian).

2. Mikhailov O.V., Stern M.B. Intrusion of porous workpieces at the openings in closed stamp. Mathematical models and descriptive experiment in materials. Kyiv: IPM named I.M.Frantsevich of NAS Ukraine. 2013, 15, pp. 8185. (in Ukrainian).

3. Beigelzimer Y.E., Varyukhin V.N., Orlov D.V., Synkov S.G. Screw extrusion is the process of strain accumulation. Donetsk: TEAN. 2003, 85 p. (in Russian).

4. Valiev R.Z., Aleksandrov I.V. Nanostructured materials obtained by intensiv plastic deformation. Moscow: Logos. 2000, 271 p. (in Russian).

5. Segal V.M., Reznikov V.I., Kopylov V.I., Pavlik D.A., Malyshev V. F. Processes of plastic structure formation of metals. Minsk: Science and technology. 1994, 232 p. (in Russian).

6. Bridgman P.V. Research of large plastic deformations and rupture. Moscow: Publishing house of foreign literature. 1955, 444 p. (in Russian).

7. Varyukhin V.N., Beygelsimer Y.Y., Synkov S.G. et al. Consolidation of amorphous A186Ni6Go2Gd6 melt spun ribbons by twist extrusion. Material Science Forum. 2006, 503-504, pp. 699-704.

8. Xiang S., Matsuki K., Tahatsuju N., Tokizawa M., Yokote T., Kusui J., Yokoi K. Microstructure and mechanical properties of PM 2024Al - 3Fe - 5Ni alloy consolidated by a new process, equal channel angular pressing. Journal of Materials Science Letters. 1997, 16, pp. 1725-1727.

9. Xia K., Wu X. Back pressure equal channel angular consolidation of pure Al particles. Scripta Materialia. 2005, 53, pp. 1225-1229.

10. Stern M.B., Serdyuk G.G. et al. Phenomenological theories of powder pressing. Kiev: Naukova Dumka. 1982, 140 p. (in Russian).

Штерн М. Б. - - чл-корр. НАН України, зав. віділом, проф. ИПМ им. И. Н. Францевича НАНУ; НТУУ «КПИ им. И. Сикорского»;

Кузьмов А. В. - канд. техн. наук, ст. науч. сотр. ИПМ им. И. Н. Францевича НАНУ; НТУУ «КПИ им. И. Сикорского»; E-mail: kavipms@bigmir.net.

ИПМ им. И. Н. Францевича НАНУ - Институт проблем материаловедения имени И. Н. Францесича Национальной академии наук Украины, г. Киев.

НТУУ «КПИ им. И. Сикорского»- Национальный технический университет Украины «Киевский политехнический институт имени I. Сикорского», г. Киев. 